\title{
Motion of a sphere in the presence of a plane interface. Part 1. An approximate solution by generalization of the method of Lorentz
}

\author{
By S. H. LEE, R. S. CHADWICK $\dagger$ \\ AND L. G. LEAL \\ Department of Chemical Engineering, California Institute \\ of Technology, Pasadena, California 91125
}

(Received 19 October 1978 and in revised form 22 February 1979)

The motion of a sphere in the presence of a fluid-fluid interface is studied. First, a solution is derived for a point force near a plane interface. Then the solution is extended to include the higher-order terms which are required to describe the motion of a solid sphere. Singularities of higher orders at the centre of the sphere are obtained by using the method of reflexions. For a fluid-fluid interface with an arbitrary viscosity ratio, the drag force and the hydrodynamic torque are calculated for the special cases of motion of a sphere perpendicular and parallel to the interface. In addition, the rotational motion of a sphere is also investigated.

\section{Introduction}

When a small solid particle moves near an interface, the drag force on the particle is changed from Stokes law due to the presence of the interface. This type of 'wall' effect plays an important role in a wide range of interesting problems including the Brownian motion of a colloidal particle, motion of micro-organisms, and the collection of small particles via bubble or drop 'flotation' type processes.

In the present paper, we study the simplest problem of this type; namely, the motion of a solid sphere in an arbitrary direction near a plane fluid-fluid interface. The Reynolds number based on particle size is assumed to be very small so that the creeping motion approximation is valid. In addition, we assume that the interface remains flat. The resulting solutions are therefore valid, as a first approximation, in any circumstances where the interface deformation remains small. Physically, this may be the case when the distance between the centre of sphere and the interface is much bigger than the sphere diameter or when either the surface tension or the density difference between the two fluids is very large.

Three distinct methods have been commonly employed to study particle motions in the presence of a flat (or nearly flat) interface; namely, (1) a standard solution via superposition using the eigensolutions of Laplace's equation in bipolar co-ordinates, (2) solution via the reciprocal theorem of Lorentz (1907), and (3) solution via the fundamental solution of Laplace's equation expressed in integral form.

The most frequently used technique, via eigensolutions of Laplace's equation in bipolar co-ordinates, was initiated by Jeffery $(1912,1915)$ who derived the general

\footnotetext{
$\dagger$ Present address: Department of Mechanics and Structures, UCLA.
} 
solution of Laplace's equation in bipolar co-ordinates, and used it to solve for the fluid motion generated by two spheres which are rotating about their line of centres. Subsequently, Stimson \& Jeffery (1926) used the same method to calculate the drag force for two spheres translating along their line of centres with the same constant velocity. Much later, Dean \& O'Neill (1963) used the general solution of Laplace's equation in bipolar co-ordinates to study the motion which is caused by the slow rotation of a sphere near an infinite rigid plane when the axis of rotation is parallel to the plane. O'Neill (1964) also investigated the translational motion of a sphere parallel to a plane solid wall. Finally, Bart (1968) extended the general solution of Jeffery (1915) for axisymmetric flow to the motion of a spherical drop which is moving normal to a liquid-liquid interface.

The reciprocal theorem approach was pioneered by Lorentz (1907) who derived a solution for the motion generated by a point force in the presence of a plane solid wall. Aderogba (1976) used the Papkovitch-Neuber solution for Stokes' equation to solve for the motion induced by a Stokeslet near a fluid-fluid interface. He superposed linear solutions with arbitrary coefficients to satisfy boundary conditions on the interface, namely the continuity of velocity and stress. However, the normal velocity at $z=0$ is not zero in his solution, which implies that the interface is not steady. Because of this, his solution is only valid at the initial instant that the stokeslet is imposed.

Finally, Faxen (1921) treated the motion of a sphere parallel to two external plane walls, using the fundamental solution of Laplace's equation in an integral form. The extension of his theory to non-spherical bodies, and to shear and parabolic flows has been carried out by many investigators (cf. Happel \& Brenner 1973).

In this paper, we generalize the reciprocal theorem approach of Lorentz (1906), to derive a general lemma for obtaining solutions of Stokes' equations that satisfy continuity of velocity, continuity of shear stress and zero normal velocity on a flat interface, given only an arbitrary solution of Stokes' equations for an unbounded domain with no interface. This lemma is then used to determine the general solution of Stokes' equations for a point force near the interface (i.e. the counterpart in this two-fluid system to the fundamental stokeslet solution in a single, unbounded fluid). Since this solution does not satisfy the continuity of normal stress, it is only valid, as indicated earlier, as a first approximation under conditions when the deformation of the interface at steady state would remain very small.

The lemma is then used to determine the motion generated by a finite size solid sphere which is translating, without rotation, either perpendicular or parallel to the interface. Provided the interface is flat, as assumed, these solutions can be superposed to obtain the solution for translation in an arbitrary direction. These solutions were obtained in the following manner. First, we put singularities at the centre of the sphere which satisfy boundary conditions in an infinite fluid (i.e. a point force and a potential dipole). Next, the lemma was used to obtain solutions for the point force and the potential dipole which satisfy boundary conditions at the interface. In general, however, these solutions do not satisfy boundary conditions at the sphere surface, and additional higher-order singularities must then be included at the centre of the sphere. The appropriate higher-order terms are determined in the asymptotic limit, $a / d \ll 1$ (where $a=$ sphere radius and $d=$ distance between sphere centre and interface), and the resulting solutions are then valid in the same limit. Similarly, we also 
studied the fluid motion generated by the rotation of a stationary sphere when the axis of rotation is parallel or perpendicular to the interface. Due to the linearity of the Stokes equation and boundary conditions, the solutions of these four problems (i.e. translation without rotation, and rotation without translation) are sufficient to determine the particle and fluid motions for any arbitrary applied force and/or torque on the particle.

The method of analysis used in this paper, and particularly the solutions for a point-force, are easily extended to the motion of slender, rod-like particles near a fluid-fluid interface. All that is required is the distribution of singularities for motion of the same particle in an infinite fluid.

The present paper comprises part 1 of a three-part study. In the forthcoming part 2 of this work, we will discuss the exact solution in bipolar co-ordinates for the motion of a sphere near a fluid-fluid interface. This exact solution is derived in the form of an infinite series, whose coefficients can be determined numerically. A detailed comparison between the approximate results of the present paper and the exact solution will be presented in part 2 . The third part of this study, part 3, is concerned with interface deformation. We consider two cases: (1) that in which the final steady-state deformation is small and calculable using the velocity and pressure fields generated in parts 1 and 2, and (2) where the interface deformation is not small. In the latter case, a numerical solution of the complete problem is required to determine the velocity and pressure fields. In part 3, we consider a novel numerical method for determining the shape of the interface in this situation. In the former case, we can use the known (i.e. calculated) shape of the interface, and the velocity fields for a flat interface to determine the first correction to the force acting on a particle due to interface deformation. This is accomplished by using the reciprocal theorem in a manner reminiscent of Ho \& Leal (1974).

\section{Basic equations}

We begin by considering the governing differential equations and boundary conditions for an arbitrarily shaped rigid particle which moves, with translational velocity $\mathbf{U}$ and angular velocity $\boldsymbol{\Omega}$, near an interface which separates two immiscible fluids that will be denoted as I and II. Apart from the disturbance flow induced by the particle, the two fluids are both assumed to be stationary. Furthermore, the undisturbed interface is assumed to be flat, and the particle to be wholly immersed in fluid II.

The theoretical analysis which follows will be valid in the limit of small Reynolds number,

$$
R e_{d}=\frac{U d}{\nu_{2}}\left(\text { or } \frac{\Omega d^{2}}{\nu_{2}}\right) \ll 1,
$$

where $\nu_{2}$ represents the kinematic viscosity of fluid II and $d$ is the separation distance between the particle 'centre' and the undisturbed interface. This condition guarantees that the particle moves a very short distance on the time scale characteristic of vorticity diffusion over the distance $d$ and, in this case, the equations of motion reduce to the steady Stokes' equations in both fluids,

$$
\left.\begin{array}{c}
0=-\nabla p_{i}+\nabla^{2} \mathbf{u}_{i}, \\
0=\nabla \cdot \mathbf{u}_{i},
\end{array}\right\} \quad i=1,2
$$


The variables in these equations may be considered to be non-dimensionalized with respect to the characteristic variables: $u_{c}=U$ (or $\Omega a$ ), $l_{c}=a$ (a particle length scale), and $p_{c}=\mu_{i} U / a$ (or $\mu_{i} \Omega$ ). In view of the linearity of these equations, and in anticipation of the fact that we will eventually restrict our attention to small deformations of the flat interface, we will consider the translational and rotational components of the particle motion separately. The boundary conditions for $\mathbf{u}_{1}$ and $\mathbf{u}_{\mathbf{2}}$ in the translational problem are thus

$$
\begin{aligned}
& \mathbf{u}_{1}, \mathbf{u}_{2} \rightarrow 0 \text { as }|\mathbf{x}| \rightarrow \infty \\
& \mathbf{u}_{2}=\mathbf{e} \text { on the particle surface; }
\end{aligned}
$$

plus the conditions

$$
\begin{aligned}
\mathbf{u}_{1} & =\mathbf{u}_{2}, \\
\mathbf{n} \cdot \mathbf{u}_{1} & =\mathbf{n} \cdot \mathbf{u}_{2}=\kappa \partial f / \partial t
\end{aligned}
$$

and

$$
\llbracket \mathbf{n} \cdot \mathbf{T} \rrbracket \equiv \lambda \mathbf{n} \cdot \mathbf{T}_{1}-\mathbf{n} \cdot \mathbf{T}_{2}=\left[\left(\frac{\sigma}{\mu_{\mathbf{2}} \bar{U}}\right)\left(-\frac{\kappa}{r} \frac{\partial f}{\partial r}-\kappa^{3} \frac{\partial^{2} f}{\partial r^{2}}\right) \mathbf{n}+\frac{g a^{2}\left(\rho_{2}-\rho_{1}\right)}{\mu_{2} \bar{U}} f \mathbf{i}_{z}\right]
$$

at the interface, $S$, which is represented by

$$
S: H \equiv z-l(t)-f(r, \phi, t)=0 .
$$

It may be noted that the dimensionless distance from the particle centre to the undeformed interface, i.e. $d(t) / a$, has been denoted as $l(t)$. The unit normal, $\mathbf{n}$, and the factor $\kappa$ are defined as

$$
\mathbf{n} \equiv \nabla H / \mid \nabla H\}, \quad \kappa \equiv 1 /|\nabla H| .
$$

The parameters appearing in $(2 e)$ are the viscosity ratio, $\lambda \equiv \mu_{1} / \mu_{2}$, the interfacial tension $\sigma$ and the density difference, $\Delta \rho \equiv \rho_{2}-\rho_{1}$, in addition to the quantities which were defined earlier. The equations $(2 c)$ and $(2 e)$ are the conditions of continuity of velocity and stress, respectively, while $(2 d)$ is the kinematic condition which relates the time rate of change of the shape function, $f$, to the normal velocities at the interface. Although we will not explicitly consider the problem of particle rotation in this initial discussion of governing equations and boundary conditions, we note here that the problem is formally identical with $U$ replaced by $\Omega$ and the condition $(2 b)$ appropriately modified to $\mathbf{u}_{2}=\hat{\mathbf{e}} \wedge \mathbf{r}$ on the particle surface.

The problem represented by (1)-(3) is, of course, both time-dependent and highly nonlinear due to the fact that $f$ is unknown. Thus, the solution (for example, the shape function $f$ ), for any instantaneous $\mathbf{U}$ and particle position (or $\boldsymbol{\Omega}$ and position plus orientation if it is non-spherical) will not be a unique function of the conditions at that instant, but rather will depend on the conditions and interface shape at earlier times. The first two papers of the present series are, however, concerned with circumstances in which the interface deformation is both small and dependent solely upon the instantaneous conditions.

The assumption that the interface shape depends only on the current conditions is, of course, precisely equivalent to the statement that the interface shape is the equilibrium shape for a given $U$ and a specified particle position; in particular, the interface shape at any instant will be the steady-state shape corresponding to the stress and pressure fields in the two fluids at that instant. Thus, the obvious physical 
requirement for this condition to be satisfied is that the particle motion be sufficiently 'slow' that the (dimensionless) particle displacement, $l^{*}$, in the time required for the interface to reach equilibrium for a given 'applied' normal stress difference, $\llbracket \mathbf{n} . \mathbf{n} . \mathbf{T} \rrbracket$, is small compared to the separation distance, $l(t)$. In addition, the time required to achieve a steady stress distribution at the interface after a change in the particle velocity (say, after an abrupt start-up of the particle motion) must be short compared to the time scale for significant displacement of the particle. The latter condition is exactly the condition $R e_{d} \ll 1$, which was already assumed in using the steady Stokes' equations. The conditions necessary in order that $l^{*} \ll l$ may be deduced by examining the equation $(2 e)$. Two distinct cases exist, depending upon whether the viscosity ratio is fixed (though perhaps large) or whether it is asymptotically large. In the latter case, the interface deformation will be small for finite times, but always unsteady and the problem is not considered in the present analysis. We consider the case in which $\lambda$ is fixed. In order to ensure that $l^{*} \ll l$ as assumed, we require either

$$
U \mu_{2} / \sigma \ll 1
$$

or

$$
U \mu_{2} / g a^{2} \Delta \rho \ll 1
$$

depending on which quantity is larger, or

$$
a / d \ll 1 .
$$

When any of the conditions (4) is satisfied, the interface deformation will not only be in quasi-equilibrium, but the magnitude of the deformation will also be asymptotically small; i.e. $O(\delta)$ in the cases $(4 a)$ or $(4 b)$ or $O\left(\delta^{n}\right)$ in case $(4 c)$, where $\delta$ represents whichever of the three parameters $(4 a-c)$ is asymptotically small and $n \geqslant 2$. The two conditions $(4 a)$ and $(4 b)$ yield a small interface deformation by balancing the normal stress jump on the left-hand side of $(2 e)$ with a large surface tension or a large density differential. The condition (4c) yields small deformations because the normal-stress difference is small, $O(a / d)^{n}$, when the particle is far from the fluid interface.

In any of these cases, the problem can be analysed completely by means of an asymptotic expansion for small $\delta$, in which

$$
f=\delta^{n} f_{1}(r, \phi)+\delta^{m+1} f_{2}(r, \phi)+\ldots
$$

and

$$
\begin{aligned}
& \mathbf{u}_{i}=\mathbf{u}_{i}^{(0)}+\delta \mathbf{u}_{i}^{(1)}+\ldots \\
& p_{i}=p_{i}^{(0)}+\delta p_{i}^{(1)}+\ldots
\end{aligned}
$$

for fixed $\lambda$. Substituting (5) and (6) into $(2 a)-(2 e)$ (for the translational case), we obtain

and, at the interface,

$$
\begin{aligned}
\mathbf{u}_{1}^{(0)}, \mathbf{u}_{2}^{(0)} \rightarrow 0 & \text { as }|\mathbf{x}| \rightarrow \infty \\
\mathbf{u}_{2}^{(0)}=\mathbf{e} & \text { on the particle surface }
\end{aligned}
$$

$$
\begin{aligned}
\mathbf{u}_{1}^{(0)} & =\mathbf{u}_{2}^{(0)}, \\
\mathbf{n} \cdot \mathbf{u}_{1}^{(0)} & =\mathbf{n} \cdot \mathbf{u}_{2}^{(0)}=0, \\
\llbracket \mathbf{t} \cdot \mathbf{n} \cdot \mathbf{T}^{(0)} \rrbracket & =0,
\end{aligned}
$$


plus one of the conditions

$$
\left.\begin{array}{ll}
\llbracket \mathbf{n} \cdot \mathbf{n} \cdot \mathbf{T}^{(0) \rrbracket}=f_{1}, & \delta \equiv \frac{\mu_{2} U}{g a^{2} \Delta \rho} \rightarrow 0, \quad \frac{\mu_{2} U}{\sigma}=O(1), \quad \frac{a}{d}=O(1) ; \\
\llbracket \mathbf{n} \cdot \mathbf{n} \cdot \mathbf{T}^{(0)} \rrbracket=-\left(\frac{1}{r} \frac{\partial f_{1}}{\partial r}+\frac{\partial^{2} f_{1}}{\partial r^{2}}\right), & \delta \equiv \frac{\mu_{2} U}{\sigma} \rightarrow 0, \quad \frac{\mu_{2} U}{g a^{2} \Delta \rho}=O(1), \quad \frac{a}{d}=O(1) ; \\
\llbracket \mathbf{n} \cdot \mathbf{n} \cdot \mathbf{T}^{(0)} \rrbracket=\left(\frac{\sigma}{\mu_{2} U}\right)\left(-\frac{1}{r} \frac{\partial f_{1}}{\partial r}-\frac{\partial^{2} f_{1}}{\partial r^{2}}\right), & \delta \equiv \frac{a}{d} \rightarrow 0, \quad \frac{\mu_{2} U}{\sigma}=O(1), \\
\frac{\mu_{2} U}{g a^{2} \Delta \rho}=O(1)+\frac{g a^{2} \Delta \rho}{\mu_{2} U} f_{1} ;
\end{array}\right\}
$$

in the limit as $\delta \rightarrow 0$. The power $m$ which appears in (5) and (6) equals 1 when $(4 a)$ or $(4 b)$ is satisfied with $a / d=O(1)$, but is equal to $n(\geqslant 2)$ for the case (4c).

The zero-order approximation, which is defined by the conditions $(7 a)-(7 e)$, plus the governing equations (1), thus represents the motion of a particle near a flat fluid interface. When the velocity and pressure fields have been determined from these equations and boundary conditions, the normal stress condition $(7 f)$ can then be used to determine a first approximation to the deviation of the interface shape from flat. Higher-order terms which account for the effects of interface deformation on the velocity and pressure fields can then be obtained via a straightforward continuation of the expansion procedure, though one must account for the interface deformation in calculating the unit normal and tangent vectors, $\mathbf{n}$ and $\mathbf{t}$, at higher order in $\delta$. In this paper, and the one (part 2) which follows, we shall be concerned only with solutions for the zero-order problem.

\section{Method of solution}

Let us then consider the solution of the equations (1), plus boundary conditions $(7 a)-(7 e)$, for the specific case of a rigid, spherical particle of radius $a$ which is immersed wholly in fluid II. As indicated in the introduction, we shall approach this problem using a generalization of the method of Lorentz (1907), which can later be adapted to solution of the same problem with more complicated particle geometry.

Lorentz (1907) used the reciprocal theorem to determine the general solution of (1) for fluid motion in the presence of a plane solid wall. We extend his solution to the general case of a fluid-fluid interface. For creeping motion of a fluid near a flat fluidfluid interface, we state the following lemma.

Lemma. Consider two immiscible fluids, fluid I for $z>0$ and fluid II for $z<0$, which are contiguous to each other at $z=0$. If $\mathbf{u}$ and $p$ are a solution of equations (1) in an infinite fluid II, then the functions

$$
\begin{aligned}
& \mathbf{u}_{1}=\frac{1}{1+\lambda}(\mathbf{u}-\hat{\mathbf{u}}), \quad p_{1}=\frac{\lambda}{1+\lambda}(p-\hat{p}) \quad \text { for } \quad z>0 \\
& \left.\begin{array}{l}
\mathbf{u}_{2}=\left(\mathbf{u}+\mathbf{u}^{*}\right)-\frac{\lambda}{1+\lambda}\left(\mathbf{u}^{*}-\hat{\mathbf{u}}^{*}\right) \\
p_{2}=\left(p+p^{*}\right)-\frac{\lambda}{1+\lambda}\left(p^{*}-\hat{p}^{*}\right)
\end{array}\right\} \text { for } z<0,
\end{aligned}
$$


satisfy equations (1), plus the conditions $(7 c-e)$ of continuity of velocity, continuity of shear stress and zero normal velocity at $z=0$. Here, $(\hat{\mathbf{u}}, \hat{p})$ is the associated solution (cf. Lorentz 1907) for $(\mathbf{u}, p)$ defined as

$$
\hat{\mathbf{u}} \equiv-\mathbf{J} \cdot \mathbf{u}-2 z \nabla w+z^{2} \nabla p
$$

and

$$
\hat{p} \equiv p+2 z \frac{\partial p}{\partial z}-4 \frac{\partial w}{\partial z}
$$

and $\left(\mathbf{u}^{*}, p^{*}\right)$ is the reflected image solution for $(\mathbf{u}, p)$ defined as

$$
\begin{aligned}
\mathbf{u}^{*} & \equiv \mathbf{J} \cdot \mathbf{u}(x, y,-z), \\
p^{*} & \equiv p(x, y,-z) .
\end{aligned}
$$

Finally, $\left(\hat{\mathbf{u}}^{*}, \hat{p}^{*}\right)$ is the associated solution for $\left(\mathbf{u}^{*}, p^{*}\right)$. The operator $\mathbf{J}$ and the constant $\lambda$ are defined as

$$
\mathbf{J} \equiv\left\{J_{i j}\right\}=\delta_{i j}-2 \delta_{i 3} \delta_{j 3}
$$

and

and $w$ is the $z$ component of $\mathbf{u}$.

$$
\lambda \equiv \mu_{1} / \mu_{2}
$$

This lemma can be easily proved by using the uniqueness of solutions for Stokes' equation (Lee 1979). $\left(\mathbf{u}_{2}, p_{2}\right)$ becomes identical to Lorentz' general solution for fluid motion near a solid wall when $\lambda$ goes to infinity. Moreover, for the points far away from the interface, $\left(\mathbf{u}_{2}, p_{2}\right)$ reduces to the solution in an infinite fluid, $(\mathbf{u}, p)$.

With the above lemma, we can easily calculate the solution of (1) for the motion of a particle in the presence of a plane interface, once the solution for particle motion in an infinite fluid is known. In the following sections, we will examine the case of a solid sphere which is translating and rotating in an arbitrary direction near an interface. Due to the linearity of equations (1), this problem can be solved by superposing solutions for translation perpendicular and parallel to the interface and for rotation with the axis of rotation perpendicular and parallel to the interface. Prior to considering this problem, however, we briefly examine the solution for a point force located in one of the fluids near a flat fluid-fluid interface.

In order to obtain this solution using our lemma, we first require the corresponding solution for a point force in a single, unbounded fluid. This is the farniliar stokeslet solution,

$$
\mathbf{u}_{s}(\mathbf{x}, \alpha)=\frac{\alpha}{R}+\frac{(\alpha \cdot \mathbf{x}) \mathbf{x}}{R^{3}}
$$

and

$$
p_{s}(\mathbf{x}, \boldsymbol{\alpha})=2 \frac{\alpha \cdot \mathbf{x}}{R^{3}}
$$

where the strength and orientation of the point force are represented by $\alpha$, and $R \equiv|\mathbf{x}|$. Let us consider a stokeslet located at $\mathbf{x}=(0,0,-l)$. Substituting equations (14) and (15) in equations (8) and (9), we obtain:

$$
\begin{aligned}
\mathbf{u}_{1, s}(\mathbf{x}, \boldsymbol{\alpha})=\frac{1}{1+\lambda}(\mathbf{I}+\mathbf{J}) \cdot( & \left.\frac{\alpha}{R_{+}}+\frac{\left(\alpha \cdot \mathbf{x}_{+}\right) \mathbf{x}_{+}}{R_{+}^{3}}\right) \\
& +\frac{2 z}{1+\lambda}\left[\frac{-\alpha_{z} \mathbf{x}_{+}}{R_{+}^{3}}+\frac{l \alpha}{R_{+}^{3}}+\frac{\left(\alpha \cdot \mathbf{x}_{+}\right)}{R_{+}^{3}} \mathbf{i}_{z}-\frac{3\left(\alpha \cdot \mathbf{x}_{+}\right) l}{R_{+}^{5}} \mathbf{x}_{+}\right]
\end{aligned}
$$




$$
\begin{gathered}
p_{1, s}(\mathbf{x}, \boldsymbol{\alpha})=\frac{-4 \lambda}{1+\lambda}\left[\frac{z \alpha_{z}}{R_{+}^{3}}-\frac{\alpha \cdot \mathbf{x}_{+}}{R_{+}^{3}}+\frac{3\left(\alpha \cdot \mathbf{x}_{+}\right) l}{R_{+}^{5}} z_{+}\right] \\
\mathbf{u}_{2, s}(\mathbf{x}, \boldsymbol{\alpha})=\mathbf{u}_{s}\left(\mathbf{x}_{+}, \boldsymbol{\alpha}\right)+\left(\frac{1}{1+\lambda} \mathbf{J}-\frac{\lambda}{1+\lambda} \mathbf{I}\right) \cdot\left(\frac{\alpha}{R_{-}}+\frac{\left(\boldsymbol{\alpha} \cdot \mathbf{x}_{-}\right)}{R_{-}^{3}} \mathbf{x}_{-}\right) \\
+\frac{2 \lambda}{1+\lambda} \frac{z}{R_{-}^{3}} \mathbf{J} \cdot\left[-\alpha_{z} \mathbf{x}_{-}+l \alpha+\left(\alpha \cdot \mathbf{x}_{-}\right) \mathbf{i}_{z}-\frac{3 l}{R_{-}^{2}}\left(\boldsymbol{\alpha} \cdot \mathbf{x}_{-}\right) \mathbf{x}_{-}\right] \\
p_{2, s}(\mathbf{x}, \boldsymbol{\alpha})=p_{s}\left(\mathbf{x}_{+}, \boldsymbol{\alpha}\right)+2 \frac{\left(\alpha \cdot \mathbf{x}_{-}\right)}{R_{-}^{3}}+4 \frac{\lambda}{1+\lambda}\left[\frac{-\alpha_{z} z}{R_{-}^{3}}-\frac{\left(\alpha \cdot \mathbf{x}_{-}\right)}{R_{-}^{3}}+\frac{3 l\left(\alpha \cdot \mathbf{x}_{-}\right) z_{-}}{R_{-}^{5}}\right]
\end{gathered}
$$

where

$$
\begin{aligned}
R_{+} & =\left(x^{2}+y^{2}+(z+l)^{2}\right)^{\frac{1}{2}}, \quad R_{-}=\left(x^{2}+y^{2}+(-z+l)^{2}\right)^{\frac{1}{2}} \\
\mathbf{x}_{+} & =(x, y, z+l), \quad \mathbf{x}_{-}=(x, y,-z+l) \\
z_{+} & =z+l, \quad z_{-}=-z+l
\end{aligned}
$$

and

$$
\mathbf{I}=\text { identity matrix, } \delta_{i j} \text {. }
$$

This solution will be used in the following sections in analysing the velocity and pressure fields generated by a finite solid sphere which is centred at $z=-l$. In addition, it may also be used in the context of a slender-body approximation to determine the velocity and pressure fields for a slender particle which is near a fluid interface.

To show the fundamental characteristics of the solution, equations (16)-(19), we will evaluate the stream function for $\alpha=\alpha_{0} \mathbf{i}_{z}$ and the normal stress imbalance at $z=0$ for $\alpha=\alpha_{0} \mathbf{i}_{z}$ and $\alpha=\alpha_{0} \mathbf{i}_{x}$. The latter are qualitatively indicative of the expected interface shape for translation of a particle normal and parallel to the undisturbed interface, $z=0$.

For the axisymmetric flow with $\alpha=\alpha_{0} \mathbf{i}_{z}$ at $z=-l$, the stream function can be easily calculated from equations (16) and (18):

$$
\begin{aligned}
& \psi_{1}=\frac{2 \alpha_{0} l z}{(1+\lambda) R_{+}}\left(1-\frac{z_{+}^{2}}{R_{+}^{2}}\right) \text { for } z>0 \\
& \psi_{2}=-\alpha_{0} R_{+}\left(1-\frac{z_{+}^{2}}{R_{+}^{2}}\right)+\alpha_{0} R_{-}\left(1-\frac{z_{-}^{2}}{R_{-}^{2}}\right)\left(1-\frac{2 \lambda}{1+\lambda} \frac{l z}{R_{-}^{2}}\right) \text { for } z<0 .
\end{aligned}
$$

In figure 1 , the stream functions for $\alpha=\frac{3}{4} \mathbf{i}_{z}$ and $\lambda=0 \cdot 01,1$, or 100 are compared with the stream function for an unbounded single fluid. It is clearly evident that the fluid motion in both fluids is retarded as the viscosity of the upper fluid increases.

The normal stress imbalance at $z=0$ can also be evaluated from the general solution, equations (16)-(19). For $\alpha=\alpha_{0} \mathbf{i}_{z}$,

and, for $\alpha=\alpha_{0} \mathbf{i}_{x}$

$$
-\Delta T_{z z}=12 \frac{\alpha_{0} l^{3}}{R_{0}^{5}}
$$

$$
-\Delta T_{z z}=12 \frac{\alpha_{0} x l^{2}}{R_{0}^{5}}
$$

Here, $R_{0} \equiv\left(l^{2}+x^{2}+y^{2}\right)^{\frac{1}{2}}$. It is noted that, for a point force located in one fluid, the normal stress imbalance on the interface is independent of viscosity ratio. In figures 2 and 3 , the right-hand sides of (22) and (23) are plotted for $\alpha_{0}=\frac{3}{4}$. It may be seen that the normal stress imbalance becomes larger as the point force approaches more closely to the interface. 


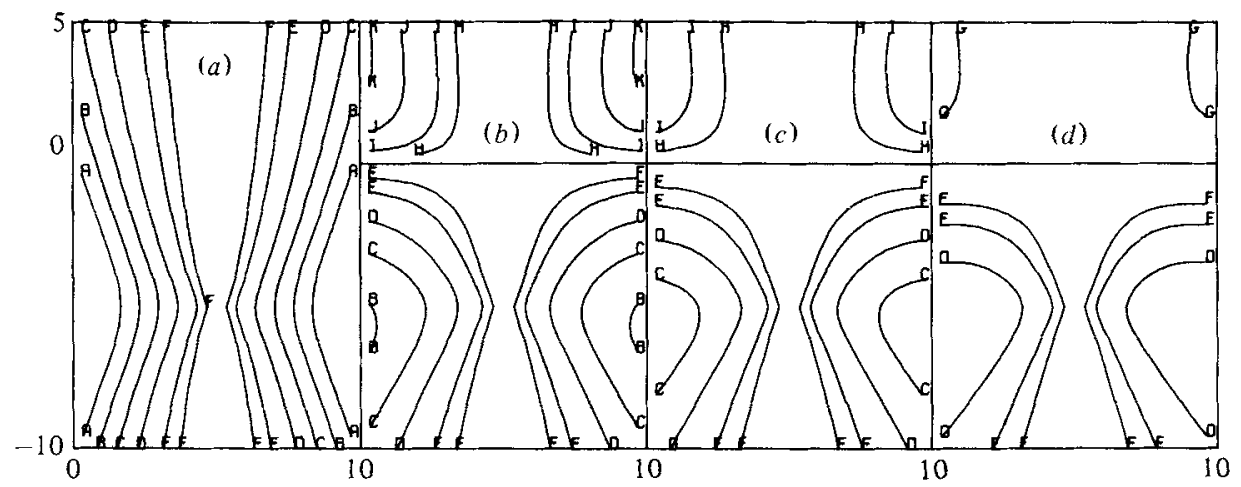

Figure 1. The streamlines due to a stokeslet, $\alpha=\frac{3}{4} \mathbf{i}_{z}\left(U_{C} l_{C}\right)$, located at $z=-5\left[l_{C}\right] . A=-2 \cdot 5$, $B=-2 \cdot 0, C=-1 \cdot 5, D=-1 \cdot 0, E=-0.5, F=-0 \cdot 25, G=0 \cdot 005, H=0 \cdot 1, I=0 \cdot 2, J=0 \cdot 4$ and $K=0 \cdot 6\left[U_{C} l_{C}^{2}\right]$. (a) Unbounded, (b) $\lambda=0 \cdot 01,(c) \lambda=1 \cdot 0,(d) \lambda=100$.

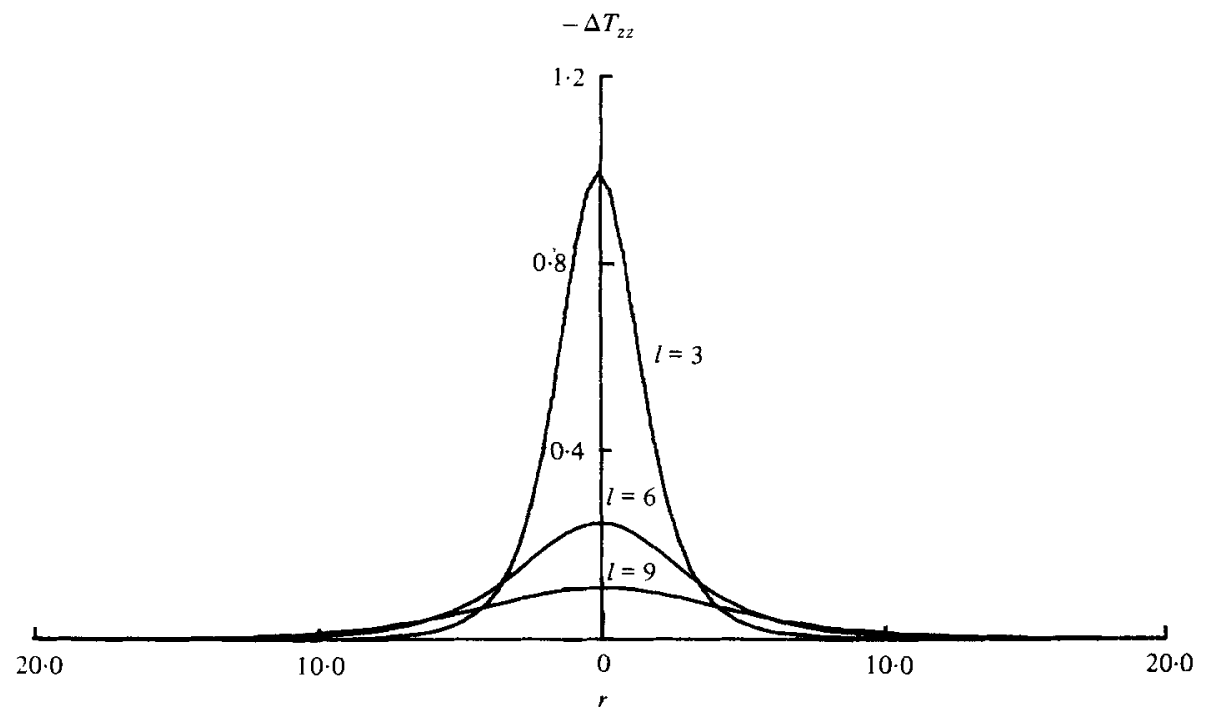

Figure 2. Normal stress imbalance on the interface, $z=0 ; \alpha=\frac{3}{4} \mathbf{i}_{z}, r=\left(x^{2}+y^{2}\right)^{\frac{1}{2}}$.

\section{Motion of a sphere normal to a plane fluid-fluid interface}

In this section, we consider the motion of a solid sphere normal to an infinite plane interface. In an infinite fluid with no external boundaries, an exact solution for translation of a solid sphere can be obtained by superposition of the fundamental solutions for a point force (i.e. the stokeslet solution) and a potential dipole, both applied at the centre of the sphere. The stokeslet solution for a point force in an infinite fluid was presented earlier, as equations (14) and (15), together with the general solution for a point force near a fluid/fluid interface which was obtained using the lemma [equations (8) and (9)] of the preceding section.

The velocity and pressure fields for a potential dipole in an infinite fluid are

$$
\mathbf{u}_{D}(\mathbf{x}, \beta)=-\frac{\beta}{R^{3}}+\frac{3(\boldsymbol{\beta} \cdot \mathbf{x}) \mathbf{x}}{R^{5}}
$$




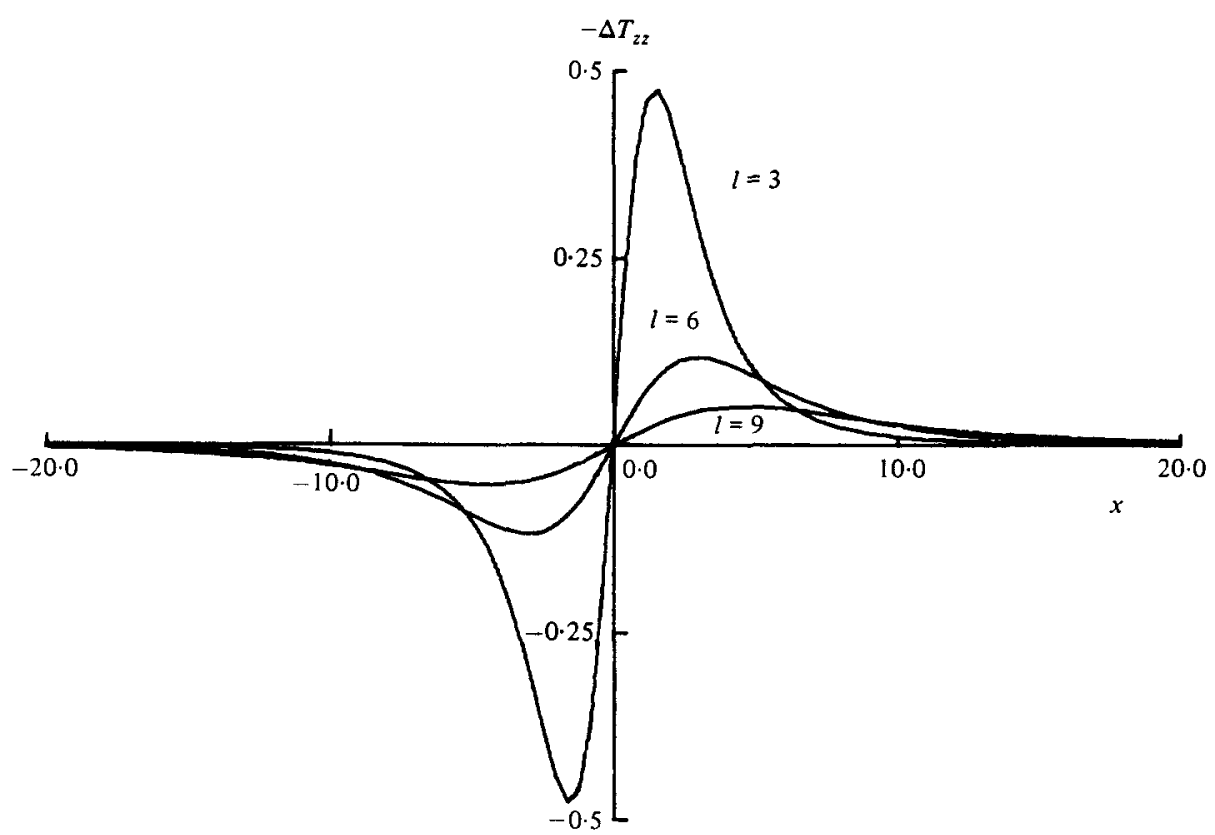

Figure 3. Normal stress imbalance on the line, $y=z=0 ; \alpha=\frac{3}{4} \mathbf{i}_{x}$.

and

$$
p_{D}(\mathbf{x}, \boldsymbol{\beta})=0
$$

where $\beta$ indicates the direction and intensity of the dipole. This solution can be generalized so that it satisfies continuity of velocity and shear stress, plus zero normal velocity, at $z=0$, by simply substituting (24) and (25) into (8) and (9). The result for a potential dipole located at $z=-l$ is

$$
\begin{aligned}
& \mathbf{u}_{1, D}(\mathbf{x}, \boldsymbol{\beta})=\frac{1}{1+\lambda}(\mathbf{I}+\mathbf{J}) \cdot\left(-\frac{\beta}{R_{+}^{3}}+\frac{3\left(\boldsymbol{\beta} \cdot \mathbf{x}_{+}\right) \mathbf{x}_{+}}{R_{+}^{5}}\right) \\
& +\frac{6 z}{1+\lambda}\left\{\frac{\beta_{z} \mathbf{x}_{+}}{R_{+}^{5}}+\frac{1}{R_{+}^{5}}\left(z_{+} \beta+\left(\boldsymbol{\beta} \cdot \mathbf{x}_{+}\right) \mathbf{i}_{z}\right)-\frac{5}{R_{+}^{7}}\left(\beta \cdot \mathbf{x}_{+}\right) z_{+} \mathbf{x}_{+}\right\} \\
& p_{1, D}(\mathbf{x}, \boldsymbol{\beta})=\frac{\lambda}{1+\lambda} \frac{12}{R_{+}^{5}}\left[2 \beta_{z} z_{+}+\beta \cdot \mathbf{x}_{+}-\frac{5}{R_{+}^{2}}\left(\boldsymbol{\beta} \cdot \mathbf{x}_{+}\right) z_{+}^{2}\right] \text {; } \\
& \mathbf{u}_{2, D}(\mathbf{x}, \boldsymbol{\beta})=u_{D}\left(\mathbf{x}_{+}, \boldsymbol{\beta}\right)+\left(\frac{1}{1+\lambda} \mathbf{J}-\frac{\lambda}{1+\lambda} \mathbf{l}\right) \cdot\left(-\frac{\beta}{R_{-}^{3}}+\frac{3\left(\boldsymbol{\beta} \cdot \mathbf{x}_{-}\right) \mathbf{x}_{-}}{R_{-}^{5}}\right) \\
& +\frac{\lambda}{1+\lambda} \frac{6 z}{R_{-}^{5}} \mathbf{J} \cdot\left\{\beta_{z} \mathbf{x}_{-}+z_{-} \beta+\left(\boldsymbol{\beta} \cdot \mathbf{x}_{-}\right) \mathbf{i}_{z}-\frac{5}{R_{-}^{2}}\left(\boldsymbol{\beta} \cdot \mathbf{x}_{-}\right) z_{-} \mathbf{x}_{-}\right\} \\
& p_{2, D}(\mathbf{x}, \beta)=-\frac{\lambda}{1+\lambda} \frac{12}{R_{-}^{5}}\left[2 \beta_{z} z_{-}+\beta \cdot \mathbf{x}_{-}-\frac{5}{R_{-}^{2}}\left(\beta \cdot \mathbf{x}_{-}\right) z_{-}^{2}\right] \text {. }
\end{aligned}
$$

The variables appearing in these solutions were all defined previously in conjunction with either (16)-(19) or (24) and (25).

Now, let us consider a sphere located with its centre at $z=-l$ which is moving with a constant velocity toward (or away from) the interface, which is located at $z=0$. In this case,

$$
\alpha=\alpha_{0}=\frac{3}{4} \mathbf{i}_{z} \text { and } \beta=\beta_{0}=-\frac{1}{4} \mathbf{i}_{z}
$$


and the zeroth-order (i.e. infinite fluid) solution can be written as

and

$$
\left.\begin{array}{l}
\mathbf{u}_{1}^{(0)}=0 \quad \text { and } \quad p_{1}^{(0)}=0, \\
\mathbf{u}_{2}^{(0)}=\mathbf{u}_{s}\left(\mathbf{x}_{+}, \frac{3}{4} \mathbf{i}_{z}\right)+\mathbf{u}_{D}\left(\mathbf{x}_{+},-\frac{1}{4} \mathbf{i}_{z}\right) \\
p_{2}^{(0)}=p_{s}\left(\mathbf{x}_{+}, \frac{3}{4} \mathbf{i}_{z}\right) .
\end{array}\right\}
$$

Here, the subscripts 1 and 2 refer to fluid I and fluid II, respectively, while the superscript (zero, in this case) indicates the level of approximation in the context of a normal reflexions-type calculation procedure.

The first correction for the presence of the interface can be evaluated simply by substituting $\alpha=\frac{3}{4} \mathbf{i}_{z}$ and $\beta=-\frac{1}{1} \mathbf{i}_{z}$ into equations (16)-(19) and (26)-(29), and subtracting the zeroth-order solution (31). We identify this first 'wall correction' by the superscript (1). For convenience, the correction corresponding to the stokeslet solution is still denoted by the subscript $s$, while that from the potential dipole is denoted by the subscript $D$, i.e.

$$
\left.\begin{array}{l}
\mathbf{u}_{1}^{(1)}=\mathbf{u}_{1, s}^{(1)}\left(\mathbf{x}, \frac{3}{4} \mathbf{i}_{z}\right)+\mathbf{u}_{1, D}^{(1)}\left(\mathbf{x},-\frac{1}{4} \mathbf{i}_{z}\right), \\
p_{1}^{(1)}=p_{1, s}^{(1)}\left(\mathbf{x}, \frac{3}{4} \mathbf{i}_{z}\right)+p_{1, D}^{(1)}\left(\mathbf{x},-\frac{1}{4} \mathbf{i}_{z}\right) ; \\
\mathbf{u}_{2}^{(1)}=\mathbf{u}_{2, s}^{(1)}\left(\mathbf{x}, \frac{3}{4} \mathbf{i}_{z}\right)+\mathbf{u}_{2, D}^{(1)}\left(\mathbf{x},-\frac{1}{4} \mathbf{i}_{z}\right), \\
p_{2}^{(1)}=p_{2,8}^{(1)}\left(\mathbf{x}, \frac{3}{4} \mathbf{i}_{z}\right)+p_{2, D}^{(1)}\left(\mathbf{x},-\frac{1}{4} \mathbf{i}_{z}\right) .
\end{array}\right\}
$$

Though $\mathbf{u}_{2}^{(0)}$ exactly satisfies the no-slip boundary condition on the surface of the sphere, additional singularities are needed at the centre of the sphere in order to cancel the velocity field correction $\mathbf{u}_{2}^{(1)}$ which is non-zero on the sphere surface. Since $\mathbf{u}_{2}^{(1)}$ is highly complicated, it is not possible to determine singularities at the sphere centre which precisely satisfy the no-slip and zero normal velocity boundary conditions. Instead, we consider the asymptotic limit $\epsilon \equiv 1 / l \ll 1$, and then choose singularities to cancel only the first few terms of $\mathbf{u}_{2}^{(1)}$ at the sphere surface, with $\mathbf{u}_{2}^{(1)}$ expressed in powers of $\epsilon$. Examination of $\mathbf{u}_{2}^{(1)}$ shows that $\mathbf{u}_{2, s}^{(1)}=O(\epsilon)$ at the sphere surface, while $\mathbf{u}_{2, D}^{(1)}$ is only $O\left(\epsilon^{3}\right)$. Hence, the dominant singularities at the next level of approximation will be those which are required to cancel the interface 'reflexion' of the point force (or stokeslet) solution. The leading terms of $\mathbf{u}_{2}^{(1)}$ near the sphere, for small $\epsilon$, are

$$
\begin{gathered}
w_{2}^{(1)}=-\epsilon \frac{\alpha_{0}}{2} \frac{2+3 \lambda}{1+\lambda}-\epsilon^{2} \frac{\alpha_{0}}{4} \frac{2+3 \lambda}{1+\lambda}(z+l)+O\left(\epsilon^{3}\right), \\
u_{2}^{(1)}=\epsilon^{2} \frac{\alpha_{0} x}{8} \frac{2+3 \lambda}{1+\lambda}+O\left(\epsilon^{3}\right), \\
v_{2}^{(1)}=\epsilon^{2} \frac{\alpha_{0} y}{8} \frac{2+3 \lambda}{1+\lambda}+O\left(\epsilon^{3}\right),
\end{gathered}
$$

with $\alpha_{0}=\left|\alpha_{0}\right|=\frac{3}{4}$.

At the first, $O(\epsilon)$, level of approximation the velocity components $u_{2}^{(1)}$ and $v_{2}^{(1)}$ parallel to the interface are zero, while the normal velocity component is simply a constant

$$
-\epsilon \frac{\alpha_{0}}{2} \frac{2+3 \lambda}{1+\lambda}
$$

Insofar as (34)-(36) are concerned, the presence of the interface is thus equivalent to an induced steady-streaming motion in the direction opposite to that of the sphere. 
Obviously, to counter $w_{2}^{(1)}$ at $O(\epsilon)$, we require a point force and a potential dipole at the sphere centre, with intensities

and

$$
\begin{aligned}
& \alpha_{1}=\epsilon \frac{3 \alpha_{0}}{8} \frac{2+3 \lambda}{1+\lambda} \mathbf{i}_{z} \\
& \beta_{1}=-\epsilon \frac{\alpha_{0}}{8} \frac{2+3 \lambda}{1+\lambda} \mathbf{i}_{z}
\end{aligned}
$$

It is important to note that the point force velocity field of strength $O(\varepsilon)$, corresponding to $\alpha_{1}$, will itself generate a vertical velocity component of $O\left(\epsilon^{2}\right)$ at the sphere surface when it is 'reflected' from the interface. Thus, if we are to consider any correction terms of $O\left(\epsilon^{2}\right)$ from (34)-(36) we must simultaneously include this additional $O\left(\epsilon^{2}\right)$ correction to the velocity field near the sphere. In order to cancel this $O\left(\epsilon^{2}\right)$ term at the sphere surface we require an additional point force and potential dipole at the sphere centre of strength

and

$$
\begin{aligned}
& \alpha_{2}=\alpha_{0}\left(\frac{3}{8} \frac{2+3 \lambda}{1+\lambda}\right)^{2} \epsilon^{2} \mathbf{i}_{z} \\
& \beta_{2}=-\frac{\alpha_{0}}{3}\left(\frac{3}{8} \frac{2+3 \lambda}{1+\lambda}\right)^{2} \epsilon^{2} \mathbf{i}_{z} .
\end{aligned}
$$

The terms of $O\left(\epsilon^{2}\right)$ in the equations (32), (33) and (34) represent an axisymmetric uniaxial extensional flow with origin at the centre of the sphere, and the $z$ axis as the symmetry axis. Chwang \& Wu (1975) have shown that an extensional flow of this general type is generated in an unbounded fluid by a stresslet and a potential quadrupole.

The basic solution of a stresslet is

$$
\begin{aligned}
& \mathbf{u}_{s s}(\mathbf{x}, \gamma, \delta)=-\left[\frac{\gamma \cdot \delta}{R^{3}}-3 \frac{(\gamma \cdot \mathbf{x})(\boldsymbol{\delta} \cdot \mathbf{x})}{R^{5}}\right] \mathbf{x}, \\
& p_{s s}(\mathbf{x}, \gamma, \delta)=-2\left[\frac{\gamma \cdot \delta}{R^{3}}-3 \frac{(\gamma \cdot \mathbf{x})(\delta \cdot \mathbf{x})}{R^{5}}\right], \\
& \omega_{s s}(\mathbf{x}, \gamma, \delta)=\frac{3}{R^{5}}[(\boldsymbol{\delta} \cdot \mathbf{x}) \gamma+(\gamma \cdot \mathbf{x}) \delta] \times \mathbf{x},
\end{aligned}
$$

where $\omega$ is the vorticity vector. The potential quadrupole is defined as the derivative of a potential dipole:

$$
\mathbf{u}_{p q}\left(\mathbf{x}, \boldsymbol{\beta}, \mathbf{i}_{l}\right)=\frac{\partial \mathbf{u}_{D}(\mathbf{x}, \boldsymbol{\beta})}{\partial x_{l}}
$$

To cancel the terms of $O\left(\epsilon^{2}\right)$ in equations (34), (35) and (36) at the sphere surface, we thus require the superposition of a stresslet and a potential quadrupole at the sphere centre. The resulting velocity field is

$$
\mathbf{u}_{2, e x}^{(2)}=\frac{1}{2} C_{1}\left[\frac{\partial}{\partial z} \mathbf{u}_{D}\left(\mathbf{x}_{+}, \mathbf{i}_{z}\right)+5 \mathbf{u}_{s s}\left(\mathbf{x}_{+}, \mathbf{i}_{z}, \mathbf{i}_{z}\right)\right]
$$

where

$$
C_{1}=\epsilon^{2} \frac{\alpha_{0}}{8} \frac{2+3 \lambda}{1+\lambda}
$$

Substituting equations (41) and (24) into (45), we obtain

$$
\mathbf{u}_{2, e x}^{(2)}=\frac{C_{1}}{2}\left[\frac{1}{R_{+}^{3}}\left\{-5 \mathbf{I}+3 \frac{2 \mathbf{I}-\mathbf{J}}{R_{+}^{2}}\right\}+15 \frac{z_{+}^{2}}{R_{+}^{5}} \mathbf{I}\left(1-\frac{1}{R_{+}^{2}}\right)\right] . \mathbf{x}_{+} .
$$


Although there is no pressure contribution from the potential quadrupole term, the contribution from the stresslet term is

$$
p_{2_{s} e x}^{(2)}=5 C_{1}\left(-\frac{1}{R_{+}^{3}}+3 \frac{z_{+}^{2}}{R_{+}^{5}}\right)
$$

Finally, the corrections to $\mathbf{u}_{2, e x}^{(2)}$ and $p_{2, e x}^{(2)}$ which are necessary to satisfy boundary conditions at the interface can be easily calculated from the lemma by substituting (46) and (47) into (8) and (9), and then subtracting (46) and (47) in fluid II. The results are:

$$
\begin{aligned}
\mathbf{u}_{1, e x}^{(3)}=\frac{C_{1}}{1+\lambda}\left[-\frac{5}{2}(\mathbf{I}\right. & +\mathbf{J}) \cdot \frac{1}{R_{+}^{3}}\left(1-3 \frac{z_{+}^{2}}{R_{+}^{2}}\right) \mathbf{x}_{+}-\frac{5 z}{R_{+}^{3}}\left(1-9 \frac{z_{+}^{2}}{R_{+}^{2}}\right) \mathbf{i}_{z} \\
& \left.+15 z\left(\frac{z_{+}}{R_{+}^{5}}-\frac{5 z_{+}^{3}}{R_{+}^{7}}\right) \mathbf{x}_{+}-15 z^{2}\left(\frac{2 \mathbf{I}-\mathbf{J}}{R_{+}^{5}}-\frac{5 z_{+}^{2} \mathbf{I}}{R_{+}^{7}}\right) \cdot \mathbf{x}_{+}\right]+O\left(\epsilon^{4}\right)
\end{aligned}
$$

and $\quad p_{1, e x}^{(3)}=\frac{C_{1} \lambda}{1+\lambda}\left(\frac{+10}{R_{+}^{3}}\right)\left[-1+3 \frac{z_{+}^{2}}{R_{+}^{2}}\left(4-\frac{5 z_{+}^{2}}{R_{+}^{2}}\right)-3 \frac{z z_{+}}{R_{+}^{2}}\left(3-5 \frac{z_{+}^{2}}{R_{+}^{2}}\right)\right]+O\left(\epsilon^{4}\right)$

in fluid I; and

$$
\begin{aligned}
\mathbf{u}_{2, e x}^{(3)}= & \frac{C_{1}}{1+\lambda}(\mathbf{J}-\lambda \mathbf{I}) \cdot \frac{5 \mathbf{x}_{-}}{2 R_{-}^{3}}\left[-1+3 \frac{z_{-}^{2}}{R_{-}^{2}}\right] \\
& +C_{1} \frac{2 \lambda}{1+\lambda} z \mathbf{J} \cdot\left(\frac{5}{2} \frac{1}{R_{-}^{3}}\right)\left[\frac{3 z_{-}}{R_{-}^{2}}\left(1-5 \frac{z_{-}^{2}}{R_{-}^{2}}\right) \mathbf{x}_{-}+\left(-1+9 \frac{z_{-}^{2}}{R_{-}^{2}}\right) \mathbf{i}_{z}\right] \\
& +C_{1} \frac{\lambda}{1+\lambda} z^{2} \mathbf{J} \cdot\left(\frac{15}{R_{-}^{5}}\right)\left[2 \mathbf{I}-\mathbf{J}-\frac{5 z_{-}^{2}}{R_{-}^{2}} \mathbf{I}\right] \cdot \mathbf{x}_{-}+O\left(\epsilon^{4}\right)
\end{aligned}
$$

and

$$
p_{2, e x}^{(3)}=\frac{5 C_{1}}{R_{-}^{3}}\left(-1+3 \frac{z_{-}^{2}}{R_{-}^{2}}\right)+\frac{C_{1} \lambda}{1+\lambda} \frac{10}{R_{-}^{3}}\left[\frac{z z_{-}}{R_{-}^{2}}\left(-9+15 \frac{z_{-}^{2}}{R_{-}^{2}}\right)-\left(\frac{12 z_{-}^{2}}{R_{-}^{2}}-15 \frac{z_{-}^{4}}{R_{-}^{4}}-1\right)\right]+O\left(\epsilon^{4}\right)
$$

in fluid II. Since the stresslet and potential quadrupole terms, (46) and (47), are $O\left(\epsilon^{2}\right)$, the 'reflected' velocity and pressure fields, $(48)-(51)$, are $O\left(\epsilon^{3}\right)$ at the sphere centre.

Hitherto, we have evaluated the singularities at the sphere centre to $O\left(\epsilon^{2}\right)$. Higherorder terms can be calculated in a similar manner, but we will not consider such terms here. In summary, the singularities required for a sphere moving perpendicularly to a plane interface are:

$$
\begin{gathered}
\mathbf{u}_{s}\left(\mathbf{x}_{+}, \frac{3}{4} \mathbf{i}_{z}\right)\left[1+\frac{3}{8} \frac{2+3 \lambda}{1+\lambda} \epsilon+\left(\frac{3}{8} \frac{2+3 \lambda}{1+\lambda}\right)^{2} \epsilon^{2}+O\left(\epsilon^{3}\right)\right] \text { stokeslet; } \\
\mathbf{u}_{D}\left(\mathbf{x}_{+},-\frac{1}{4} \mathbf{i}_{z}\right)\left[1+\frac{3}{8} \frac{2+3 \lambda}{1+\lambda} \epsilon+\left(\frac{3}{8} \frac{2+3 \lambda}{1+\lambda}\right)^{2} \epsilon^{2}+O\left(\epsilon^{3}\right)\right] \text { potential dipole; } \\
\epsilon^{2} \frac{5 \alpha_{0}}{16} \frac{2+3 \lambda}{1+\lambda} \mathbf{u}_{s 8}\left(\mathbf{x}_{+}, \mathbf{i}_{z}, \mathbf{i}_{z}\right) \quad \text { stresslet; }
\end{gathered}
$$

and

$$
\epsilon^{2} \frac{\alpha_{0}}{16} \frac{2+3 \lambda}{1+\lambda} \frac{\partial \mathbf{u}_{D}}{\partial z}\left(\mathbf{x}_{+}, \mathbf{i}_{z}\right) \text { potential quadrupole. }
$$




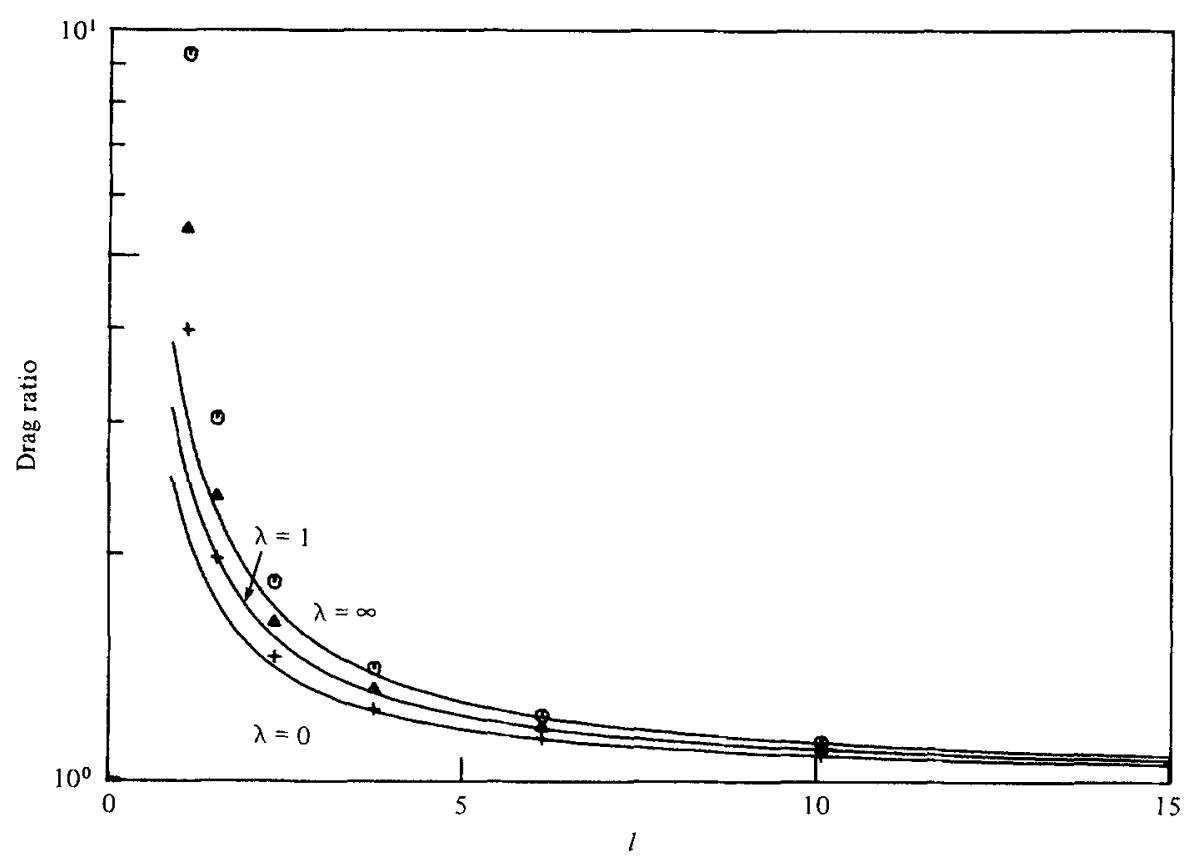

Figure 4. The drag ratio vs. the distance between the sphere centre and the interface; $\mathbf{U}=\mathbf{i}_{\mathbf{z}} . \odot, \lambda=\infty ; \Delta, \lambda=1 ;+, \lambda=0 ;$ Bart (1968). $\longrightarrow$, equation (52).

As there is no contribution to the drag force from a potential dipole, stresslet or potential quadrupole, the drag ratio (the drag divided by Stokes' drag) is simply given as

$$
\text { drag ratio }=1+\frac{3}{8} \epsilon \frac{2+3 \lambda}{1+\lambda}+\left(\frac{3}{8} \epsilon \frac{2+3 \lambda}{1+\lambda}\right)^{2}+O\left(\epsilon^{3}\right) .
$$

When $\lambda \rightarrow \infty$, equation (52) reduces to the drag ratio for the case of solid wall, and is identical with Wakiya's results to $O\left(\epsilon^{2}\right)$ (cf. Happel \& Brenner 1973).

In figure 4 , the drag ratio, equation (52), is plotted versus the distance between the sphere centre and the interface for $\lambda=0,1$ and $\infty$. The drag ratios numerically calculated by Bart (1968) are also shown in the figure. As previously mentioned, we presume $\epsilon \ll 1$ in the derivation of (52). Thus, for $\epsilon \ll 1,(52)$ agrees with Bart's result which is the exact solution for a sphere motion near a flat interface. Even for $l \sim 2 \cdot 5$, the approximate solution shows reasonably good agreement with the exact solution. However, the two solutions deviate from each other as the sphere approaches more closely to the interface. Since the convergence of $(52)$ is poor for $\epsilon \approx 1$, we need higherorder terms if our expansion technique is to be used for $l<2 \cdot 5$. Even then, close agreement would not be expected for $\epsilon \equiv l^{-1} \approx 1$ since the exact solution is singular in that limit.

\section{Motion of a sphere parallel to a plane fluid-fluid interface}

Let us now turn to the problem of a non-rotating sphere which is translating parallel to a plane fluid-fluid interface. The zeroth-order solution for this problem can be obtained by substituting $\alpha=\alpha_{0} \mathbf{i}_{x}$ and $\beta=-\frac{1}{3} \alpha_{0} \mathbf{i}_{x}$ into equations (30) and (31), with $\mathbf{u}_{s}$ and $\mathbf{u}_{D}$ evaluated from (14), (15), (24) and (25). Similarly, the first correction 
by reflexion from the interface can be obtained from equations (32) and (33), with (16)-(19) and (26)-(29).

The no-slip boundary condition on the sphere is not satisfied since the 'reflected' flow field is non-zero at the sphere surface. We may examine the leading terms of this reflected field, expressed as a power series in $\epsilon$ :

$$
\begin{aligned}
& u_{2}^{(1)}=\epsilon \frac{\alpha_{0}}{4} \frac{2-3 \lambda}{1+\lambda}+\epsilon^{2} \frac{\alpha_{0}}{8} \frac{2-3 \lambda}{1+\lambda}(z+l)+O\left(\epsilon^{3}\right) ; \\
& v_{2}^{(1)}=O\left(\epsilon^{3}\right) \\
& w_{2}^{(1)}=-\epsilon^{2} \frac{\alpha_{0} x}{8} \frac{3 \lambda+2}{1+\lambda}+O\left(\epsilon^{3}\right) .
\end{aligned}
$$

In order to satisfy the no-slip and zero normal velocity conditions at the sphere surface, we need additional singularities at the sphere centre which produce the velocity field of opposite sign. For the term of $O(\epsilon)$, a stokeslet and a potential dipole are required, which have the intensity and orientation

and

$$
\alpha_{1}=-\epsilon \frac{3 \alpha_{0}}{16} \frac{2-3 \lambda}{1+\lambda} \mathbf{i}_{x}
$$

By induction, we also know that the interface 'reflexion' of the point force and potential dipole solutions corresponding to (56) and (57) will yield a non-zero contribution of $O\left(\epsilon^{2}\right)$ to the $x$ component of velocity at the sphere surface. In order to satisfy boundary conditions on the sphere surface to $O\left(\epsilon^{2}\right)$, we thus require an additional point force and potential dipole at the sphere centre with magnitude and orientation

and

$$
\begin{aligned}
& \alpha_{2}=\epsilon^{2} \alpha_{0}\left(\frac{3}{16} \frac{2-3 \lambda}{1+\lambda}\right)^{2} \mathbf{i}_{x} \\
& \beta_{2}=-\epsilon^{2} \frac{\alpha_{0}}{3}\left(\frac{3}{16} \frac{2-3 \lambda}{1+\lambda}\right)^{2} \mathbf{i}_{x} .
\end{aligned}
$$

Further, we require singularities to cancel the $O\left(\epsilon^{2}\right)$ contributions in (53) and (55). Examination shows that these terms represent a linear shear flow with origin at the sphere centre. Chwang \& Wu (1975) discovered that a stresslet, a rotlet and a potential quadrupole were necessary to produce such a flow in an unbounded fluid. Thus, the velocity field which cancels the terms of $O\left(\epsilon^{2}\right)$ in $\mathbf{u}^{(1)}$ at the sphere is

$$
\begin{aligned}
\mathbf{u}_{2, S H}^{(2)}=C_{2}\left\{\frac{5}{6} \mathbf{u}_{s s}\left(\mathbf{x}_{+}, \mathbf{i}_{x}, \mathbf{i}_{z}\right)\right. & \left.+\frac{1}{2} \mathbf{u}_{R}\left(\mathbf{x}_{+}, \mathbf{i}_{y}\right)+\frac{1}{6} \frac{\partial}{\partial z} \mathbf{u}_{D}\left(\mathbf{x}_{+}, \mathbf{i}_{x}\right)\right\} \\
& +C_{3}\left\{\frac{5}{6} \mathbf{u}_{s s}\left(\mathbf{x}_{+}, \mathbf{i}_{z}, \mathbf{i}_{x}\right)-\frac{1}{2} \mathbf{u}_{R}\left(\mathbf{x}_{+}, \mathbf{i}_{y}\right)+\frac{1}{6} \frac{\partial}{\partial x} \mathbf{u}_{D}\left(\mathbf{x}_{+}, \mathbf{i}_{z}\right)\right\} .
\end{aligned}
$$

Here,

$$
C_{2}=-\frac{\epsilon^{2} \alpha_{0}}{8} \frac{2-3 \lambda}{1+\lambda}, \quad C_{3}=\frac{\epsilon^{2} \alpha_{0}}{8} \frac{3 \lambda+2}{1+\lambda},
$$

and $\mathbf{u}_{R}$ is the velocity field due to a rotlet defined as

$$
\mathbf{u}_{R}(\mathbf{x}, \boldsymbol{\gamma})=\boldsymbol{\gamma} \times \mathbf{x} / R^{3} \text {. }
$$


Substituting (24), (41) and (61) into (60) yields

$$
\begin{aligned}
\mathbf{u}_{2, S H}^{(2)}=C_{2}[ & \left.\frac{z_{+}}{2}\left(\frac{1}{R_{+}^{5}}+\frac{1}{R_{+}^{3}}\right) \mathbf{i}_{x}+\frac{1}{2} x\left(\frac{1}{R_{+}^{5}}-\frac{1}{R_{+}^{3}}\right) \mathbf{i}_{z}+\frac{5}{2} x z_{+}\left(\frac{1}{R_{+}^{5}}-\frac{1}{R_{+}^{7}}\right) \mathbf{x}_{+}\right] \\
& +C_{3}\left[\frac{z_{+}}{2}\left(\frac{1}{R_{+}^{5}}-\frac{1}{R_{+}^{3}}\right) \mathbf{i}_{x}+\frac{1}{2} x\left(\frac{1}{R_{+}^{5}}+\frac{1}{R_{+}^{3}}\right) \mathbf{i}_{z}+\frac{5}{2} x z_{+}\left(\frac{1}{R_{+}^{5}}-\frac{1}{R_{+}^{7}}\right) \mathbf{x}_{+}\right] .
\end{aligned}
$$

The pressure becomes

$$
p_{2, S H}^{(2)}=5\left(C_{2}+C_{3}\right) \frac{x z_{+}}{R_{+}^{5}}
$$

The corrections (i.e. reflexions) to $\left(\mathbf{u}_{2, S H}^{(2)}, p_{2, S H}^{(2)}\right)$ which are necessary to satisfy boundary conditions at the interface can be calculated easily from the lemma. The results are

$$
\begin{aligned}
& \mathbf{u}_{1, S H}^{(3)}=\frac{1}{1+\lambda}(\mathbf{I}+\mathbf{J}) \cdot \frac{1}{2 R_{+}^{3}}\left[C_{4}\left(z_{+} \mathbf{i}_{x}-x \mathbf{i}_{z}\right)+5 C_{5} \frac{x z_{+}}{R_{+}^{2}} \mathbf{x}_{+}\right] \\
& +\frac{z}{R_{+}^{3}}\left[-C_{4}+5 C_{5} \frac{z_{+}^{2}}{R_{+}^{2}}\right] \mathbf{i}_{x}-5 z^{2} C_{5} \frac{1}{R_{+}^{5}}\left(-5 \frac{x z_{+}}{R_{+}^{2}} \mathbf{x}_{+}+z_{+} \mathbf{i}_{x}+x \mathbf{i}_{z}\right) \\
& +\frac{x z}{R_{+}^{5}}\left[\left(3 C_{4}-25 C_{5} \frac{z_{+}^{2}}{R_{+}^{2}}\right) \mathbf{x}_{+}+10 C_{5} z_{+} \mathbf{i}_{z}\right]+O\left(\epsilon^{4}\right), \\
& p_{1, S H}^{(3)}=\frac{\lambda}{1+\lambda} \frac{x}{R_{+}^{5}}\left[10 C_{5} z\left(\frac{5 z_{+}^{2}}{R_{+}^{2}}-1\right)+20 C_{5} z_{+}+6 C_{4} z_{+}-50 C_{5} \frac{z_{+}^{3}}{R_{+}^{2}}\right]+O\left(\epsilon^{5}\right) ; \\
& \mathbf{u}_{2, S H}^{(3)}=\left(\frac{1}{1+\lambda} \mathbf{J}-\frac{\lambda}{1+\lambda} \mathbf{l}\right) \cdot\left[\frac{1}{2 R_{-}^{3}} C_{4}\left(z_{-} \mathbf{i}_{x}-x \mathbf{i}_{z}\right)+\frac{5}{2} C_{5} \frac{x z_{-}}{R_{-}^{5}} \mathbf{x}_{-}\right] \\
& +\frac{\lambda z}{1+\lambda} \frac{\mathbf{J}}{R_{-}^{3}}\left[C_{4}\left(-\mathbf{i}_{x}+\frac{3 x \mathbf{x}_{-}}{R_{-}^{2}}\right)+\frac{5 C_{5} z_{-}}{R_{-}^{2}}\left(z_{-} \mathbf{i}_{x}+2 x \mathbf{i}_{z}-\frac{5 x z_{-} \mathbf{x}_{-}}{R_{-}^{2}}\right)\right] \\
& +\frac{5 \lambda}{1+\lambda} z^{2} \mathbf{J} \cdot C_{5}\left[-\frac{5}{R_{-}^{2}} x z_{-} \mathbf{x}_{-}+\frac{1}{R_{-}^{5}}\left(z_{-} \mathbf{i}_{x}+x \mathbf{i}_{z}\right)\right]+O\left(\epsilon^{4}\right), \\
& p_{2, S H}^{(3)}=5 C_{5} \frac{x z_{-}}{R_{-}^{5}}-\frac{2 \lambda}{1+\lambda}\left[5 z C_{5}\left(\frac{-5 x z_{-}^{2}}{R_{-}^{7}}+\frac{x}{R_{-}^{5}}\right)+3 C_{4} \frac{x z_{-}}{R_{-}^{5}}-25 C_{5} \frac{x z_{-}^{3}}{R_{-}^{7}}+10 C_{5} \frac{x z_{-}}{R_{-}^{5}}\right]+O\left(\epsilon^{5}\right) \text {. } \\
& C_{4}=C_{2}-C_{3}=-\epsilon^{2} \frac{\alpha_{0}}{2} \frac{1}{1+\lambda} \\
& C_{5}=C_{2}+C_{3}=\epsilon^{2} \frac{3 \alpha_{0}}{4} \frac{\lambda}{1+\lambda} .
\end{aligned}
$$

Thus, for a sphere moving parallel to an interface, the singularities required at the centre of sphere through $O\left(\epsilon^{2}\right)$ are:

$$
\begin{gathered}
\mathbf{u}_{s}\left(\mathbf{x}_{+}, \frac{3}{4} \mathbf{i}_{x}\right)\left\{1-\epsilon \frac{3}{16} \frac{2-3 \lambda}{1+\lambda}+\left(\epsilon \frac{3}{16} \frac{2-3 \lambda}{1+\lambda}\right)^{2}+O\left(\epsilon^{3}\right)\right\} \quad \text { stokeslet; } \\
\mathbf{u}_{D}\left(\mathbf{x}_{+},-\frac{1}{4} \mathbf{i}_{x}\right)\left\{1-\epsilon \frac{3}{16} \frac{2-3 \lambda}{1+\lambda}+\left(\epsilon \frac{3}{16} \frac{2-3 \lambda}{1+\lambda}\right)^{2}+O\left(\epsilon^{3}\right)\right\} \text { potential dipole; } \\
\frac{5}{6} C_{5} \mathbf{u}_{s s}\left(\mathbf{x}_{+}, \mathbf{i}_{x}, \mathbf{i}_{z}\right) \quad \text { stresslet; } \\
\frac{1}{2} C_{4} \mathbf{u}_{R}\left(\mathbf{x}_{+}, \mathbf{i}_{y}\right) \quad \text { rotlet; } \\
\frac{1}{6}\left(C_{2} \frac{\partial}{\partial z} \mathbf{u}_{D}\left(\mathbf{x}_{+}, \mathbf{i}_{x}\right)+C_{3} \frac{\partial}{\partial x} \mathbf{u}_{D}\left(\mathbf{x}_{+}, \mathbf{i}_{z}\right)\right) \text { potential quadrupole. }
\end{gathered}
$$




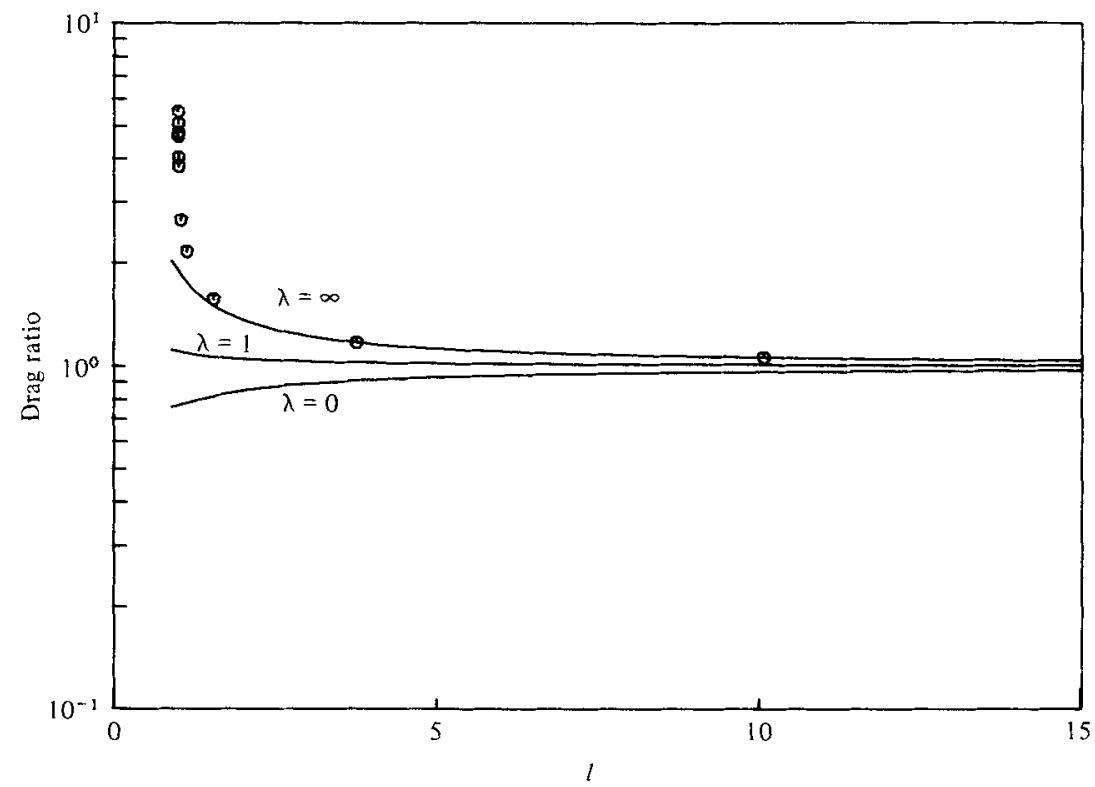

Figure 5. The drag ratio vs. the distance between the sphere centre and the interface; $\mathbf{U}=\mathbf{i} x . \odot, \lambda=\infty$ (O'Neill 1964); —., equation (68).

We can evaluate the drag ratio easily from the stokeslet strength,

$$
\text { drag ratio }=1-\epsilon \frac{3}{16} \frac{2-3 \lambda}{1+\lambda}+\epsilon^{2}\left(\frac{3}{16} \frac{2-3 \lambda}{1+\lambda}\right)^{2}+O\left(\epsilon^{3}\right) .
$$

It can be seen that there exists a critical viscosity ratio equal to $\frac{2}{3}$, above which the drag force on the sphere in the presence of an interface is larger than that in an infinite fluid. For $\lambda<\frac{2}{3}$, the drag is less than it would be in an infinite fluid. Further, equation (68) becomes identical to Faxen's (1921) results to $O\left(\epsilon^{2}\right)$ when $\lambda$ goes to infinity.

From the rotlet strength, the hydrodynamic torque on the particle can be obtained and is equal to

$$
\mathbf{T}=-8 \pi \mathbf{i}_{y} \frac{1}{2}\left(C_{2}-C_{3}\right)+O\left(\epsilon^{3}\right)=\frac{3 \pi}{2} \epsilon^{2} \frac{1}{1+\lambda} \mathbf{i}_{y}+O\left(\epsilon^{3}\right)
$$

This is the negative of the torque which is required to keep the particle from rotating. It will be noted that, when $\lambda \rightarrow \infty$, there is no term of $O\left(\epsilon^{2}\right)$ in $\mathbf{T}$. Indeed, Faxen's (1921) solution for sphere motion near a solid wall yields $\mathbf{T} \sim O\left(\epsilon^{\mathbf{4}}\right)$. It is also noteworthy that for any finite $\lambda$ the sense of the applied torque for a sphere which is far from the interface is opposite in direction to that which might be expected intuitively, and opposite to that which is required for a sphere near a solid wall (see part 2 of this work).

In figure 5, the drag ratio, equation (68), is plotted versus $l$, the distance between the sphere centre and the interface, for $\lambda=0,1$, and $\infty$. O'Neill (1964) calculated the drag ratio for the motion of a sphere parallel to a plane solid wall by using bipolar co-ordinates. His results are also shown in figure 5. There is a good agreement between the two solutions in the region of $\epsilon \ll 1$. When a sphere approaches the interface, we need more higher-order terms in equation (68) due to the poor convergence of equation (68) in $\epsilon$ power series. As expected, the difference between equation (68) and O'Neill's solution becomes larger as $\epsilon \rightarrow 1$. 


\section{Rotation of a sphere in the presence of an interface}

Finally, we turn to the case of a stationary sphere rotating with a constant angular velocity $\gamma$ in the presence of a plane interface. $\gamma$ is non-dimensionalized with respect to $u_{c} / l_{c}$. The solution in an infinite fluid can be simply represented by a rotlet at the centre of sphere, and the velocity field generated by a rotlet is given by equation (61). Thus the zeroth-order solution for a rotating sphere in the presence of a plane interface is

$$
\begin{aligned}
& \mathbf{u}_{1}^{(0)}=0 ; \\
& \mathbf{u}_{2}^{(0)}=\mathbf{u}_{R}\left(\mathbf{x}_{+}, \boldsymbol{\gamma}\right) .
\end{aligned}
$$

The first correction by reflexion from the interface can be easily obtained from the lemma;

$$
\begin{gathered}
\mathbf{u}_{1}^{(1)}=\frac{(\mathbf{I}+\mathbf{J})}{1+\lambda} \cdot\left(\frac{\gamma \times \mathbf{x}_{+}}{R_{+}^{3}}\right)+\frac{2 z}{1+\lambda}\left[\frac{\left(\gamma_{x} \mathbf{i}_{y}-\gamma_{y} \mathbf{i}_{x}\right)}{R_{+}^{3}}-3 \mathbf{x}_{+} \frac{\left(\gamma_{x} y-\gamma_{y} x\right)}{R_{+}^{5}}\right] \\
\mathbf{u}_{2}^{(1)}=\left(\frac{1}{1+\lambda} \mathbf{J}-\frac{\lambda}{1+\lambda} \mathbf{I}\right) \cdot \frac{\left(\gamma \times \mathbf{x}_{-}\right)}{R_{-}^{3}}+\frac{2 \lambda}{1+\lambda} z\left[\frac{\left(\gamma_{x} \mathbf{i}_{y}-\gamma_{y} \mathbf{i}_{x}\right)}{R_{-}^{3}}-3 \mathbf{J} \cdot \mathbf{x}_{-} \frac{\left(\gamma_{x} y-\gamma_{y} x\right)}{R_{-}^{5}}\right] .
\end{gathered}
$$

Here, $\gamma_{x}, \gamma_{y}$ and $\gamma_{z}$ are the components of $\gamma$ in $\mathbf{i}_{x}, \mathbf{i}_{y}$ and $\mathbf{i}_{z}$ directions, respectively.

Since the problem is linear, the solution for rotation with an arbitrary axis of rotation can be obtained by superposition of the solutions for rotation when this axis is parallel and normal, respectively, to the interface. Therefore, without loss of generality, we will solve only these two problems for rotation of a sphere.

First, let us consider a rotating sphere when the axis of rotation is perpendicular to the interface (i.e. $\gamma_{z} \neq 0, \gamma_{x}=\gamma_{y}=0$ ). As discussed in the previous sections, the first correction (73) to the solution due to the presence of the interface does not satisfy no-slip boundary conditions at the sphere surface. Hence, we analyse the leading terms of (73) at the sphere surface as a power series in $\epsilon$,

$$
\begin{gathered}
u_{2}^{(1)}=-\frac{1-\lambda}{1+\lambda} \frac{\gamma_{z}}{8} \epsilon^{3} y+O\left(\epsilon^{4}\right), \\
v_{2}^{(1)}=\frac{1-\lambda}{1+\lambda} \frac{\gamma_{z}}{8} \epsilon^{3} x+O\left(\epsilon^{4}\right)
\end{gathered}
$$

and

$$
w_{2}^{(1)}=0 \text {. }
$$

To cancel this additional velocity field at the sphere surface, a rotlet is needed at the centre of sphere. The velocity field generated by this rotlet is given by

$$
\mathbf{u}_{2}^{(2)}=-\frac{1}{8} \frac{1-\lambda}{1+\lambda} \epsilon^{3} \mathbf{u}_{R}\left(\mathbf{x}_{+}, \gamma_{z} \mathbf{i}_{z}\right)
$$

Consequently, for the rotation of a sphere whose axis is normal to the interface, only a rotlet is required at the centre of sphere through terms of $O\left(\epsilon^{3}\right)$ and its strength is as follows:

$$
\mathbf{u}_{i}\left(\mathbf{x}_{+}, \gamma_{z} \mathbf{i}_{z}\right)\left(1-\frac{1-\lambda}{8(1+\lambda)} \epsilon^{3}+O\left(\epsilon^{4}\right)\right) \quad \text { rotlet. }
$$

The torque required to maintain angular velocity, $\gamma=\gamma_{z} \mathbf{i}_{z}$, can be easily calculated from the strength of rotlet,

$$
\mathbf{T}=8 \pi \gamma_{z}\left(1-\frac{1-\lambda}{8(1+\lambda)} \epsilon^{3}+O\left(\epsilon^{4}\right)\right) \mathbf{i}_{z} .
$$


For $\lambda>1$, the required torque on the sphere in the presence of an interface is larger than that in an infinite fluid while, for $\lambda<1$, it is smaller. It may be noted in this case that there is no contribution to the drag force up to $O\left(\epsilon^{3}\right)$. As expected, rotation about the $z$ axis induces no translational motion of the particle.

Now, let us consider a rotating sphere whose rotation axis is parallel to a plane interface. Substituting $\gamma_{x} \neq 0$ and $\gamma_{y}=\gamma_{z}=0$ into equation (73) and expanding each term in the power series of $\epsilon$, we can evaluate the leading terms of equation (73) at the sphere surface:

$$
\begin{aligned}
& u_{2}^{(1)}=O\left(\epsilon^{4}\right) \\
& v_{2}^{(1)}=-\frac{1}{4} \frac{\gamma_{x}}{1+\lambda} \epsilon^{2}-\frac{2-\lambda}{8(1+\lambda)} \gamma_{x}(l+z) \epsilon^{3}+O\left(\epsilon^{4}\right) ; \\
& w_{2}^{(1)}=-\frac{1+4 \lambda}{8(1+\lambda)} \gamma_{x} y \epsilon^{3}+O\left(\epsilon^{4}\right) .
\end{aligned}
$$

In order to counter the term of $O\left(\epsilon^{2}\right)$ in $v_{2}^{(1)}$, we need a stokeslet and a potential dipole at the centre of sphere with strength and orientation

and

$$
\boldsymbol{\alpha}_{1}=\epsilon^{2} \frac{3}{16} \frac{\gamma_{x}}{1+\lambda} \mathbf{i}_{y}
$$

In addition, for the shear flows of $O\left(\epsilon^{3}\right)$ in equations (80) and (81), a stresslet, a rotlet and a potential quadrupole are required at the centre of sphere. They yield the following velocity field:

$$
\mathbf{u}_{2, S H}^{(2)}=\frac{3}{8} \gamma_{x} \epsilon^{3}\left[\frac{5}{6} \mathbf{u}_{s s}\left(\mathbf{x}_{+}, \mathbf{i}_{y}, \mathbf{i}_{z}\right)+\frac{1}{6} \frac{\partial}{\partial y} \mathbf{u}_{D}\left(\mathbf{x}_{+}, \mathbf{i}_{z}\right)\right]+\frac{1}{16} \frac{-1+5 \lambda}{1+\lambda} \gamma_{x} \epsilon^{3} \mathbf{u}_{R}\left(\mathbf{x}_{+}, \mathbf{i}_{x}\right)
$$

Hence, the rotational motion of a sphere whose rotation axis is parallel to an interface requires the following singularities at the centre of sphere:

$$
\begin{gathered}
\mathbf{u}_{R}\left(\mathbf{x}_{+}, \gamma_{x} \mathbf{i}_{x}\right)\left[1+\frac{-1+5 \lambda}{1+\lambda} \frac{\epsilon^{3}}{16}+O\left(\epsilon^{4}\right)\right] \text { rotlet; } \\
\mathbf{u}_{s}\left(\mathbf{x}_{+}, \mathbf{i}_{y}\right)\left[\frac{3}{16} \frac{\gamma_{x}}{1+\lambda} \epsilon^{2}+O\left(\epsilon^{3}\right)\right] \quad \text { stokeslet; } \\
\mathbf{u}_{D}\left(\mathbf{x}_{+}, \mathbf{i}_{y}\right)\left[-\frac{1}{16} \frac{\gamma_{x}}{1+\lambda} \epsilon^{2}+O\left(\epsilon^{3}\right)\right] \quad \text { potential dipole; } \\
\mathbf{u}_{s s}\left(\mathbf{x}_{+}, \mathbf{i}_{y}, \mathbf{i}_{z}\right) \frac{5}{16} \gamma_{x} \epsilon^{3} \quad \text { stresslet; } \\
\frac{\partial}{\partial y} \mathbf{u}_{D}\left(\mathbf{x}_{+}, \mathbf{i}_{z}\right) \frac{1}{16} \gamma_{x} \epsilon^{3} \quad \text { potential quadruple. }
\end{gathered}
$$

The torque on the sphere required to achieve angular velocity, $\gamma=\gamma_{x} \mathbf{i}_{x}$, can be readily evaluated from the strength of rotlet:

$$
\mathbf{T}=8 \pi \gamma_{x}\left[1+\frac{-1+5 \lambda}{1+\lambda} \frac{\epsilon^{3}}{16}+O\left(\epsilon^{4}\right)\right] \mathbf{i}_{y}
$$


It can be seen that, for $\lambda>\frac{1}{5}$, the required torque on the sphere increases due to the presence of an interface. For $\lambda<\frac{1}{5}$, the torque is less than it would be in an infinite fluid. In addition, there will be a drag force on the sphere which can be obtained from the strength of the stokeslet as

$$
\mathbf{F}=-\frac{3 \pi}{2} \frac{\gamma_{x}}{1+\lambda} \epsilon^{\mathbf{2}} \mathbf{i}_{y}+O\left(\epsilon^{3}\right)
$$

In this case, rotation will lead to translation of the sphere parallel to the interface unless a body force of equal magnitude and opposite sign is applied to the sphere. For a solid wall, $\lambda \rightarrow \infty$, there is no contribution to the drag force up to $O\left(\epsilon^{2}\right)$ from the rotational motion of a sphere.

\section{Discussion}

In the previous sections, we have studied the translational and rotational motions of a sphere in the presence of a fluid-fluid interface. Due to the linearity of Stokes' equation, we can analyse the arbitrary motion of a sphere in the presence of an interface by superposing the translation of a sphere parallel and perpendicular to the interface and the rotation of a sphere with the axis of rotation parallel or perpendicular to the interface. Hence, an arbitrary motion of a sphere can be expressed in more general terms; namely, in terms of a translation tensor, a rotation tensor and a coupling tensor.

At sufficiently small Reynolds numbers, the motion of a rigid particle can be generally described by [cf. Happel \& Brenner 1973]:

$$
\begin{aligned}
& \mathbf{F}=\mathbf{K}_{T} \cdot \mathbf{U}+\mathbf{K}_{C}^{+} \cdot \boldsymbol{\Omega} ; \\
& \mathbf{T}=\mathbf{K}_{C} \cdot \mathbf{U}+\mathbf{K}_{R} \cdot \mathbf{\Omega} .
\end{aligned}
$$

Here, $\mathbf{F}$ and $\mathbf{T}$ are the force and the torque exerted on the particle, $\mathbf{K}_{T}, \mathbf{K}_{R}$ and $\mathbf{K}_{C}$ are the translation tensor, the rotation tensor, and the coupling tensor, respectively. $\mathbf{U}$ is the translational velocity of a particle and $\boldsymbol{\Omega}$ is the angular velocity.

Using the results in $\$ \S 4-6$, the elements of the tensors, $\mathbf{K}_{T}, \mathbf{K}_{R}$ and $\mathbf{K}_{C}$, can be determined for motion of a sphere near a plane fluid-fluid interface, viz.

$\mathbf{K}_{T}=6 \pi\left[\begin{array}{lll}K_{\|}^{T} & & 0 \\ & K_{\|}^{T} & \\ 0 & & K_{\perp}^{T}\end{array}\right], \quad \mathbf{K}_{R}=8 \pi\left[\begin{array}{lll}K_{\sharp}^{R} & & 0 \\ & K_{\|}^{R} & \\ 0 & & K_{\perp}^{R}\end{array}\right], \quad \mathbf{K}_{C}=6 \pi\left[\begin{array}{ccc}0 & K_{C} & 0 \\ -K_{C} & 0 & 0 \\ 0 & 0 & 0\end{array}\right]$,

where

$$
\boldsymbol{K}_{\mathrm{i}}^{T}=1-\frac{3}{16} \epsilon \frac{2-3 \lambda}{1+\lambda}+\left(\frac{3}{16} \epsilon \frac{2-3 \lambda}{1+\lambda}\right)^{2}+O\left(\epsilon^{3}\right),
$$

$$
\begin{aligned}
& K_{\perp}^{T}=1+\frac{3}{8} \epsilon \frac{2+3 \lambda}{1+\lambda}+\left(\frac{3}{8} \epsilon \frac{2+3 \lambda}{1+\lambda}\right)^{2}+O\left(\epsilon^{3}\right), \\
& K_{\sharp}^{R}=1+\frac{\epsilon^{3}}{16} \frac{-1+5 \lambda}{1+\lambda}+O\left(\epsilon^{4}\right),
\end{aligned}
$$$$
K_{\perp}^{R}=1-\frac{\epsilon^{3}}{8} \frac{1-\lambda}{1+\lambda}+O\left(\epsilon^{4}\right)
$$

and

$$
K_{C}=\frac{\epsilon^{2}}{4} \frac{1}{1+\lambda}+O\left(\epsilon^{3}\right) \text {. }
$$


Since we have completed the evaluation of the resistance tensors in equations (87) and (88), we can easily solve other interesting problems. As one example, the motion of a freely suspended sphere under the action of an applied $\mathbf{F}$ can be obtained by substituting $\mathbf{T}=0$ in equation (88). The translational and angular velocities for this case are

and

$$
\begin{gathered}
\mathbf{U}=\left[\mathbf{K}_{T}-\mathbf{K}_{C}^{+} \cdot \mathbf{K}_{R}^{-1} \cdot \mathbf{K}_{C}\right]^{-1} \cdot \mathbf{F} \\
\mathbf{\Omega}=-\mathbf{K}_{\boldsymbol{R}}^{-1} \cdot \mathbf{K}_{C} \cdot \mathbf{U} .
\end{gathered}
$$

\section{Conclusions}

We have derived the creeping flow solution for a point force in the presence of an interface with an arbitrary viscosity ratio. In addition, we have generalized this solution to the case of a finite-size sphere moving near a fluid-fluid interface. In the limit of an infinite viscosity ratio, these results are in accord with known solutions for motion of a sphere near a plane solid wall.

When a sphere moves normal to a plane interface, we have shown that it is necessary to modify the strength of the stokeslet and potential dipole singularities at the sphere centre, as well as add a stresslet and a potential quadrupole through terms of $O\left(\epsilon^{2}\right)$. The drag ratio is

$$
\left(1+\frac{3}{8} \epsilon \frac{2+3 \lambda}{1+\lambda}+\left(\frac{3}{8} \epsilon \frac{2+3 \lambda}{1+\lambda}\right)^{2}\right)
$$

For the case of a sphere moving parallel to the interface, on the other hand, it is necessary to include a rotlet, as well as the stokeslet, potential dipole, potential quadrupole and stresslet. In this case, the drag ratio is

$$
\left(1-\frac{3}{1 \overline{6}} \epsilon \frac{2-3 \lambda}{1+\lambda}+\left(\frac{3}{1 \overline{6}} \epsilon \frac{2-3 \lambda}{1+\lambda}\right)^{2}\right) \text {. }
$$

In addition, we have shown that a torque

$$
\frac{3 \pi}{2} \epsilon^{2} \frac{1}{1+\lambda}
$$

is required to keep the particle from rotating. It can be seen that the drag on a sphere moving normal to the interface is increased relative to Stokes' drag for all values of $\epsilon$ and $\lambda$. This is a reflection of the presence of a flat interface. Even when the upper fluid becomes essentially inviscid relative to the lower fluid (i.e. $\lambda \rightarrow 0$ ), the drag on the particle is increased. The additional dissipation in fluid II which is associated with the interface is obviously greater than the decrease in dissipation in fluid I as $\lambda \rightarrow 0$. For parallel motion and $\epsilon \ll 1$, on the other hand, there is a critical viscosity ratio $\lambda=\frac{2}{3}$ which separates the regions of increased and decreased drag.

We have also studied the rotation of a sphere with a constant angular velocity near an interface. When the rotation axis is normal to the interface, only a rotlet is needed at the centre of sphere through terms of $O\left(\epsilon^{3}\right)$ and the hydrodynamic torque on the sphere surface is proportional to

$$
\left(1-\frac{1-\lambda}{8(1+\lambda)} \epsilon^{3}\right)
$$

There is no drag force for this case. 
When a sphere is rotating with axis of rotation parallel to the interface, a rotlet as well as a stokeslet, a potential dipole, a potential quadrupole and a stresslet is required at the centre of sphere. The required hydrodynamic torque is proportional to

$$
\left(1-\frac{1-5 \lambda}{1+\lambda} \frac{\epsilon^{3}}{16}\right)
$$

Further, a force,

$$
\frac{3 \pi}{2} \frac{\gamma_{x}}{1+\lambda} \epsilon^{2}
$$

is required to keep the sphere from translating from its original position. The critical viscosity ratio which separates the regions of increased and decreased torque due to the presence of an interface is 1 for the rotation with a normal axis and $\frac{1}{5}$ for the rotation with a parallel axis.

In conclusion, we can solve for arbitrary motions of a sphere in the presence of a plane interface by linearly superposing the approximate solutions for translational and rotational motions of a sphere.

The solution scheme which we have developed can be applied, in principle, for the motion of an arbitrary body near a flat interface, provided only that we know the singularity distribution in an infinite fluid. However, it should be mentioned that the higher-order terms are valid only when $\epsilon \ll 1$.

This work was supported by a grant, ENG78-10317, from the National Science Foundation. The authors also wish to thank Professor Howard Brenner for many helpful discussions during the initial stages of this work.

\section{REFERENCES}

Aderogba, K. 1976 On Stokeslets in a two-fluid space. J. Eng. Math. 10, 143.

BART, E. 1968 The slow unsteady settling of a fluid sphere toward a flat fluid interface. Chem. Eng. Sci. 23, 193.

Chwang, A. T. \& WU, T. Y. 1975 Hydromechanics of low-Reynolds number flow. Part 2. Singularity method for Stokes flow. J. Fluid Mech. 67, 787.

Dean, W. R. \& O'Neill, M. E. 1963 A slow motion of viscous liquid caused by the rotation of a solid sphere. Mathematika 10,13.

Faxen, H. 1921 Dissertation, Uppsala University.

HAPPel, J. \& BRenner, H. 1973 Low Reynolds Number Hydrodynamics. Noordhoff International Publishers.

Ho, B. P. \& Leal, L. G. 1974 Inertial migration of rigid spheres in two-dimensional unidirectional flows. J. Fluid Mech. 65, 365.

JEFFERY, G. B. 1912 On a form of the solution of Laplace's equation suitable for problems relating to two spheres. Proc. Roy. Soc. A 87, 109.

JEFFERY, G. B. 1915 On the steady rotation of a solid of revolution in a viscous fluid. London Math. Soc. 14, 327.

LEE, S. H. 1979 Ph.D. Dissertation, California Institute of Technology.

Lonentz, H. A. 1907 A general theory concerning the motion of a viscous fluid. Abhandl. Theoret. Phys, 1, 23.

O'NeILl, M. E. 1964 A slow motion of viscous liquid caused by a slowly moving solid sphere. Mathematika 11, 67.

Stimson, M. \& Jeffery, G. B. 1926 The motion of two spheres in a viscous fluid. Proc. Roy. Soc. A 111, 110. 